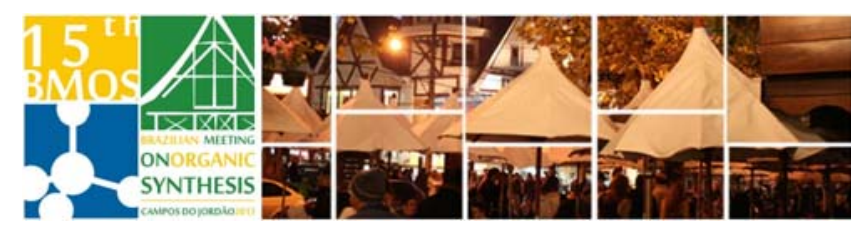

\title{
Catalysis and mechanistic investigation with ionically-tagged reagents
}

\author{
Prof. Dr. B. A. D. Neto
}

Laboratory of Medicinal and Technological Chemistry, University of Brasilia - Chemistry Institute - IQ-UnB, Campus Universitário Darcy Ribeiro, CEP 70904-970 - P.O.Box 4478 - Brasília - DF - Brazil

*e-mail: brenno.ipi@gmail.com

Keywords: ionically-tagged catalysts, biomimetic transformations, ESI-MS(/MS)

\section{Abstract Speech}

This flash presentation focus on the concept, development, synthesis and application of new ionically-tagged catalysts applied for cross-coupling, multicomponent reactions, oxidation and reduction reactions. The ionic tags are responsible for the nanoorganisation and nano- effect observed for those new catalysts. The presence of at least one ionic tag allows the direct investigation of the reactions mechanisms via electrospray (tandem) mass spectrometry - ESI-MS(/MS) - with important implications for the biomimetic transformations compared with the enzymatic ones (e.g. Cytochrome P-450 oxidation). The catalysts could be recycled and reused for several times without any notable loss of catalytic activity.

\section{REFERENCES}

${ }^{1}$ Curtis, M. D.: Shiu, K.; Butler, W. M. e Huffmann, J. C. J. Am. Chem. Soc 1986, 108, 3335.

${ }^{2}$ Curtis, M. D.; Shiu, K.; Butler, W. M. e Huffmann, J. C. J. Am. Chem. Soc. 1986, 108, 3335 . 